Check for updates

Cite this: RSC Adv., 2018, 8, 40589

Received 8th October 2018

Accepted 16th November 2018

DOI: $10.1039 / c 8 r a 08293 f$

rsc.li/rsc-advances

\section{In situ sulfuration synthesis of flexible PAN-CuS "flowering branch" heterostructures as recyclable catalysts for dye degradation $\uparrow$}

\author{
Yin Lu, ${ }^{a}$ Yanjie Wang, ${ }^{a}$ Shizhong Cui, ${ }^{a}$ Weihua Chen (DD *b and Liwei Mi (DD *a \\ "Flowering branch"-like PAN-CuS hierarchical heterostructures were in situ synthesized through a facile \\ hydrothermal sulfuration growth process on PAN-based fibers prepared by electrospinning. The PAN \\ fibers can serve as a stable flexible support, while CuS flowers assembled from nanosheets can act as \\ reactive materials, showing high performance in the degradation of dyes. Moreover, these \\ heterostructures can be recovered easily without a decrease in their photocatalytic activity, thus showing \\ favorable recycling capability.
}

"Green hill and clear water" is not only a "China dream", but a shared vision of the whole world. As issues related to the environment are drawing increasing attention, many researchers have focused on the degradation of pollutants in industrial wastewater. ${ }^{1-3}$ Dyestuff wastewater accounts for a large proportion of industrial wastewater, which is featured by complicated organic matter composition, high density and toxicity. ${ }^{4-6}$ In recent years, dye degradation technologies have been developed, mainly including adsorption, ozonation, and electrochemical and photochemical degradation..$^{7-9}$ Physical methods cannot resolve the problem extensively and are apt to cause secondary pollution. Biochemical methods have high selectivity, but their technology is complex. Through photocatalytic oxidation, the organic macromolecular pollutants can be oxidized directly or indirectly to non-polluting molecules, such as $\mathrm{CO}_{2}$ and $\mathrm{H}_{2} \mathrm{O}^{10,11}$ This technology also has the advantages of mild operating conditions, significant degrading effects, thorough purification, and a lack of secondary pollution. Photocatalytic oxidation is expected to be one of the most effective treatments. Many previous studies have shown that metal oxide/chalcogenide semiconductors play an important part in the photocatalytic process. ${ }^{12-16}$ However, for now, there are still challenges regarding the large-scale construction of recyclable photocatalytic materials through mild methods.

Metal oxide/chalcogenide semiconductors have the outstanding attributes of unique optical, electronic, magnetic

${ }^{a}$ Center for Advanced Materials Research, Zhongyuan University of Technology, Zhengzhou, 450007, P. R. China. E-mail: mlwzzu@163.com

${ }^{b}$ College of Chemistry and Molecular Engineering, Zhengzhou University, Zhengzhou, 450001, P. R. China. E-mail: chenweih@zzu.edu.cn

$\dagger$ Electronic supplementary information (ESI) available: The experimental section, SEM images of PAN-Cu ${ }^{2+}$ and PAN-CuS (Fig. S1), SEM images of CuS on pure PAN fibers (Fig. S2), EDX spectrum of PAN-CuS-1, PAN-CuS-3 (Fig. S3), SEM images of PAN-CuS after recycle (Fig. S4). See DOI: 10.1039/c8ra08293f and thermal properties as well as potential applications in energy conversion and catalysis, particularly in the field of photocatalysis. ${ }^{17-20}$ Recently, copper sulfides have attracted a great deal of attention owing to their widespread applications in solar cells, optical filters, photoelectric transformers, superconductors and sensors. ${ }^{21-23}$ Particularly, covellite (CuS) has a strong p-type metallic character with the highest concentration of free carriers among copper sulfide materials. ${ }^{24}$ Many previous researchers have demonstrated that CuS has important applications for photocatalysis. For instance, Saranya et al. prepared a CuS catalyst to treat organic pollutants, showing a degradation time of over 60 min. $^{25}$ Thuy et al. reported CuS and $\mathrm{CuS} / \mathrm{ZnS}$ core/shell nanocrystals for photocatalytic degradation of dyes under visible light, and showed that the pollutants almost bleached over a period of $2 \mathrm{~h}^{26}$ Varieties of $\mathrm{CuS}$ micro- and nanostructures. ${ }^{27-35}$ have been synthesized using different methods including solid-state synthesis, solvothermal solution based methods, sacrificial templating and chemical vapor deposition techniques..$^{28,30,36-40,44}$ Similar to most reports, CuS has mainly been synthesized in powder form, which is not convenient for recycling. For developing recyclable photocatalytic material, it is important to explore the large-sale preparation methods with low cost. It is known that electrospinning is a powerful method to fabricate one-dimensional functional materials with large specific surface-areas, high porosity, good flexibility and high stability. ${ }^{41-43}$

Herein, we elaborately developed stringed "flowering branch" PAN-CuS with hierarchical architecture combining fibrous PAN skeleton and active CuS material. First, we obtained PAN-Cu ${ }^{2+}$ composite nanofibers from a simple electrospinning method. Then, the PAN-CuS composite nanostructures were prepared through a further in situ sulfidizing process. Then, the degradation activity for the dye pollutants and recyclability of the as-synthesized PAN-CuS nanostructures were studied. The 


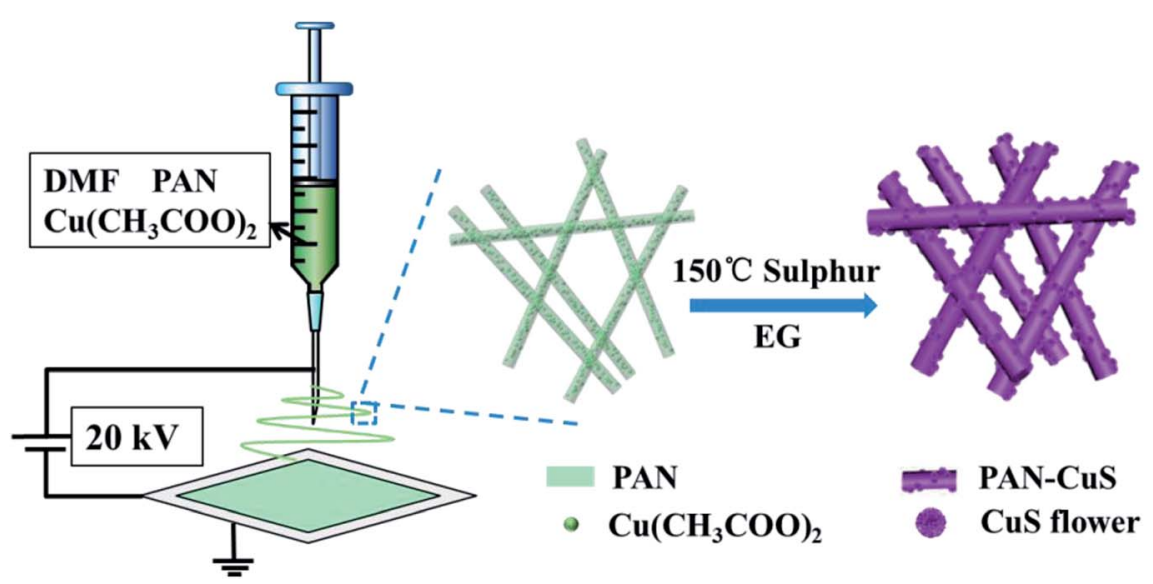

Fig. 1 Schematic of the synthesis of PAN-CuS hierarchical heterostructures.

superior photocatalytic activity can be attributed to the stable fibrilous structure and abundant active sites of the CuS nanoflowers assembled from nanosheets. Fig. 1 illustrates the synthesis process of the PAN-CuS hierarchical heterostructures.

To explore the effects of different amounts of $\mathrm{Cu}^{2+}, 1 \mathrm{mmol}$, $1.5 \mathrm{mmol}$, or $2 \mathrm{mmol} \mathrm{Cu}\left(\mathrm{CH}_{3} \mathrm{COO}\right)_{2}$ was added to PAN/DMF to synthesize PAN-Cu-1, PAN-Cu-2, PAN-Cu-3 nanofilms, respectively. Fig. S1a and $\mathrm{b} \dagger$ show the SEM images of the PAN-Cu-2 nanofibers. The nanofiber has a diameter of about $500 \mathrm{~nm}$ and length of several tens of micrometers, with a continuous interlacing network. After sulfidation, $\mathrm{Cu}^{2+}$ ions move to the surface to produce CuS nanoflowers, and the nanofiber structure is well-maintained to function as the primary structure. As shown in Fig. S1c and $d, \dagger$ the diameter of the CuS nanoflower is about $200 \mathrm{~nm}$, and is assembled from nanosheets, thus possessing abundant active sites. A previous study ${ }^{\mathbf{4 4}}$ reported the formation process as follows:

$$
\begin{gathered}
2 \mathrm{HOCH}_{2} \mathrm{CH}_{2} \mathrm{OH}-2 \mathrm{H}_{2} \mathrm{O} \rightarrow 2 \mathrm{CH}_{3} \mathrm{CHO} \rightarrow 2 \mathrm{H}_{3} \mathrm{CCOCOCH}_{3} \\
\mathrm{~S}+2 \mathrm{H} \rightarrow \mathrm{H}_{2} \mathrm{~S} \\
\mathrm{Cu}^{2+}+\mathrm{H}_{2} \mathrm{~S} \rightarrow \mathrm{CuS}+2 \mathrm{H}^{+}
\end{gathered}
$$

The reaction temperature was chosen to be $150{ }^{\circ} \mathrm{C}$ to exceed the melting point of sulfur $\left(120^{\circ} \mathrm{C}\right)$. First, acetaldehyde can be generated by the dehydration of EG at high temperatures, where acetaldehyde can donate a hydrogen atom and act as a reducing agent, as shown in eqn (1). Then, $\mathrm{S}^{2-}$ ions are formed through the reduction of $\mathrm{S}$ by $\mathrm{H}$ atoms, as shown in eqn (2). When the PAN film came in contact with $\mathrm{EG}$, the $\mathrm{Cu}^{2+}$ ions move from the inside to the surface and then form the CuS crystal nucleus. When the ion concentration was moderate, the crystal nucleus grew into nanosheets and further self-assembled into nanoflowers. As shown in Fig. 2a-f, for PAN-CuS-1 and PAN-CuS-2, more $\mathrm{Cu}^{2+}$ corresponds to more CuS flowers. However, as $\mathrm{Cu}^{2+}$ further increased, the CuS of the PAN-CuS-3 mainly showed the morphology of nanosheets. This phenomenon was proposed to be attributed to the increased CuS nucleation number with high $\mathrm{Cu}^{2+}$ content; this will result in a lower crystal growth process, thus limiting the formation of $\mathrm{CuS}$ nanoflowers. ${ }^{45}$ In addition, we prepared CuS on pure PAN fibers for comparison. The SEM image is shown in Fig. S2.† Compared with in situ synthesis of CuS on PAN-Cu ${ }^{2+}$ fibers, Fig. S2 $\uparrow$ shows that CuS flowers are very uneven, indicating the advantage of the in situ sulfuration method of PAN-Cu ${ }^{2+}$ films.

The XRD pattern of PAN-CuS-2 films (Fig. 2g) shows a broad diffraction peak at around $24.9^{\circ}$, which is attributed to the electrospun nanofibers. ${ }^{\mathbf{4 6 7}}$ Other diffraction peaks can be well assigned to CuS (JCPDS no. 6-464). No additional peaks for other phases were observed, indicating the high purity of CuS. The EDX spectrum (Fig. 2h) further confirms the presence of C, $\mathrm{O}, \mathrm{Cu}$ and $\mathrm{S}$ elements in the hierarchical nanostructures. The EDX spectra of PAN-CuS-1, PAN-CuS-3 are also shown in Fig. S3† for understanding the components of these samples. The microstructure was further examined using transmission electron microscopy (TEM). The TEM image in Fig. 2i clearly demonstrates the heterostructure of the PAN-CuS composite. The CuS flower is assembled by nanosheets with an average length of about $50 \mathrm{~nm}$. It is expected that these CuS nanosheets possess relative more active sites. The high magnification TEM image in Fig. $2 \mathrm{j}$ shows the lattice pattern of the CuS nanoparticle and reveals a lattice spacing of $0.321 \mathrm{~nm}$, which is in a good agreement with the interplanar distance of (101) of hexagonal CuS. The selected-area electron diffraction pattern (Fig. 2j inset) indicates that the CuS nanoparticles are polycrystalline. As shown in Fig. S4, $\dagger$ FT-IR spectroscopy was performed to identify the components of the nanocomposites. As shown in the FT-IR spectrum, the characteristic peak at $1072.28 \mathrm{~cm}^{-1}$ can be attributed to the $\mathrm{S}=\mathrm{O}$ and $\mathrm{C}-\mathrm{C}$ stretching vibration, while the vibration peaks at $1359.91 \mathrm{~cm}^{-1}$ and $1450.46 \mathrm{~cm}^{-1}$ are associated with the $\mathrm{CH}_{3}$ and $\mathrm{CH}_{2}$ stretching vibrations, respectively. The peak at $1730.24 \mathrm{~cm}^{-1}$ can be attributed to the $\mathrm{C}=\mathrm{O}$ stretching vibration. The peak at $2241.64 \mathrm{~cm}^{-1}$ is attributed to the stretching vibration of $\mathrm{C} \equiv \mathrm{N}$. The peaks at $2934.24 \mathrm{~cm}^{-1}$ can be attributed to the $\mathrm{C}-\mathrm{H}$ stretching vibration in $\mathrm{CH}_{3}$. Furthermore, the presence of vibrational peaks at $612.71 \mathrm{~cm}^{-1}$ indicates the presence of $\mathrm{Cu}-\mathrm{S}$ 

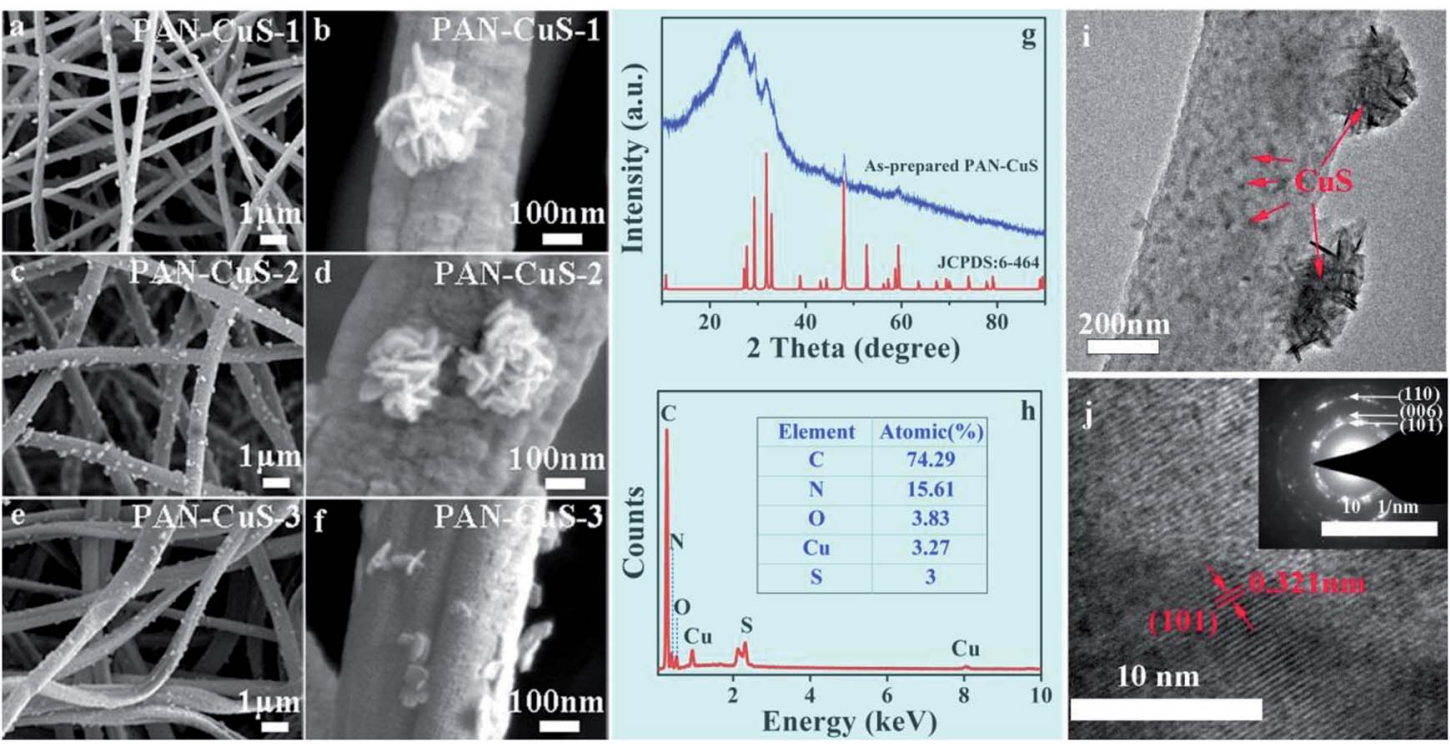

Fig. 2 (a-f) SEM images of different PAN-CuS hierarchical heterostructures. (a and b) PAN-CuS-1. (c and d) PAN-CUS-2. (e and f) PAN-CuS-3. (g) XRD diffraction patterns of PAN-CuS-2 hierarchical heterostructures. (h) A typical EDX spectrum of the PAN-CuS-2 hierarchical heterostructures. (i) TEM image of PAN-CuS-2 hierarchical heterostructures; (j) HRTEM image of the heterojunction region and SAED of the nanosheet (inset).

stretching modes. The surface chemistry of the as-developed PAN-CuS film was studied by XPS measurement. In Fig. S5a, $\uparrow$ the wide-scan XPS spectrum indicated the presence of $\mathrm{Cu}$ and $\mathrm{S}$ from the CuS nanoparticles as well as $\mathrm{C}, \mathrm{N}$, and $\mathrm{O}$ from the electrospun PAN nanofibers and other organic solvents. Fig. S5b $\dagger$ represents a high-resolution spectrum of $\mathrm{Cu} 2 \mathrm{p}$. The peaks for $\mathrm{Cu}$ indicated the typical $\mathrm{Cu} 2 \mathrm{p} 3 / 2(932.30 \mathrm{eV})$ and $\mathrm{Cu}$ $2 \mathrm{p} 1 / 2(952.80 \mathrm{eV})$ binding energies. The XPS spectrum of $\mathrm{S} 2 \mathrm{p}$ in Fig. S5c $\dagger$ is characterized by peaks at 162.5 and $163.2 \mathrm{eV}$. At the same time, the $\mathrm{C}$ peak centered at $285 \mathrm{eV}$ could be assigned to the bonds of $\mathrm{C}-\mathrm{C}(284.6 \mathrm{eV}), \mathrm{C}-\mathrm{O}(285.6 \mathrm{eV})$, and $\mathrm{O}-\mathrm{C}=\mathrm{O}(289$ $\mathrm{eV})$, demonstrating the presence of the carboxyl carbon.

We explored the catalytic properties of different samples: pure PAN film, PAN-Cu ${ }^{2+}$ film, and PAN-CuS film. All the tests were conducted in the presence of $\mathrm{H}_{2} \mathrm{O}_{2}$ under UV light.

Fig. 3a shows the absorption spectra of aqueous solutions of MB tested at different intervals in the presence of the CuS (PANCuS-2) architectures. The intense absorption peak at $664 \mathrm{~nm}$ of MB decreased gradually with the prolonged irradiation time, indicating the degradation of MB. The decoloring degree of aqueous MB reached $46.2 \%, 80.7 \%, 93.6 \%$, and $97.1 \%$ after 15 , $30,45,50 \mathrm{~min}$, respectively. This good catalytic performance can be attributed to the unique features of the hierarchical nanostructures: fibrous skeleton, transition metal sulfide, abundant active sites, high porosity and strong connection between $\mathrm{CuS}$ and electrospun nanofibers. When $\mathrm{H}_{2} \mathrm{O}_{2}$ was added, photoelectrons were consumed, thus preventing photonic electrons from recombining with holes, which improved quantum efficiency. Fig. $3 \mathrm{~b}$ exhibits the degradation activity for different $\mathrm{Cu}$ content. For PAN-CuS-1, the decoloring degree was $37.6 \%, 77.0 \%, 92.4 \%$, and $94.6 \%$ in $15,30,45$, $50 \mathrm{~min}$, respectively. For PAN-CuS-3, the decoloring degree was $57.9 \%, 86.1 \%, 94.6 \%$, and $96.7 \%$ in $15,30,45$, and $50 \mathrm{~min}$, respectively. As the $\mathrm{Cu}$ content increased, the degradation rate increased. To further study the degradation kinetics, the first order rate constants for the degradation of MB (Fig. 3c) were calculated to be $0.07032 \mathrm{~min}^{-1}, 0.08035 \mathrm{~min}^{-1}$, and $0.07099 \mathrm{~min}^{-1}$. The first order rate constant is described as follows:

$$
\ln \left(C_{0} / C_{t}\right)=k t
$$

where $k$ is the apparent rate constant, $C_{0}$ is the original concentration of $\mathrm{MB}$, and $C_{t}$ is the equilibrium concentration of $\mathrm{MB}$ at the relative reaction time. The calculated results show that the PAN-CuS-2 film has the highest $k$ among the samples. As mentioned above, more CuS nanoflowers lead to better catalytic properties, indicating that CuS indeed plays a decisive role. Fig. 3d systematically displays $\mathrm{MB}$ degradation activity after 30 min for different samples. When adding only $\mathrm{H}_{2} \mathrm{O}_{2}$ without PAN-CuS catalyst, the degradation of MB was only $17 \%$, and the pure PAN film also had a mild effect on MB due to its adsorption capacity (the degradation degree was about $45 \%$ ). However, the pure PAN film corroded after one cycle and could not be reused. For PAN-Cu ${ }^{2+}$ and PAN-CuS, the degradation of MB was $70 \%$ and $83 \%$, respectively, which demonstrates the advantage of the in situ sulfuration method of PAN-Cu films. Fig. 3e also displays the MB degradation activity by plotting $C_{t} /$ $C_{0}$ as a function of time; the samples were the same as those in Fig. 3d. Thus, the good photocatalytic performance of PAN-CuS hierarchical heterostructures is due to the following factors. First, the nanosheet structure of CuS not only allows more surfaces to receive the incident light, but also exhibits more active catalytic sites, which results in a good photocatalytic performance. Second, the as-adopted fabrication route 

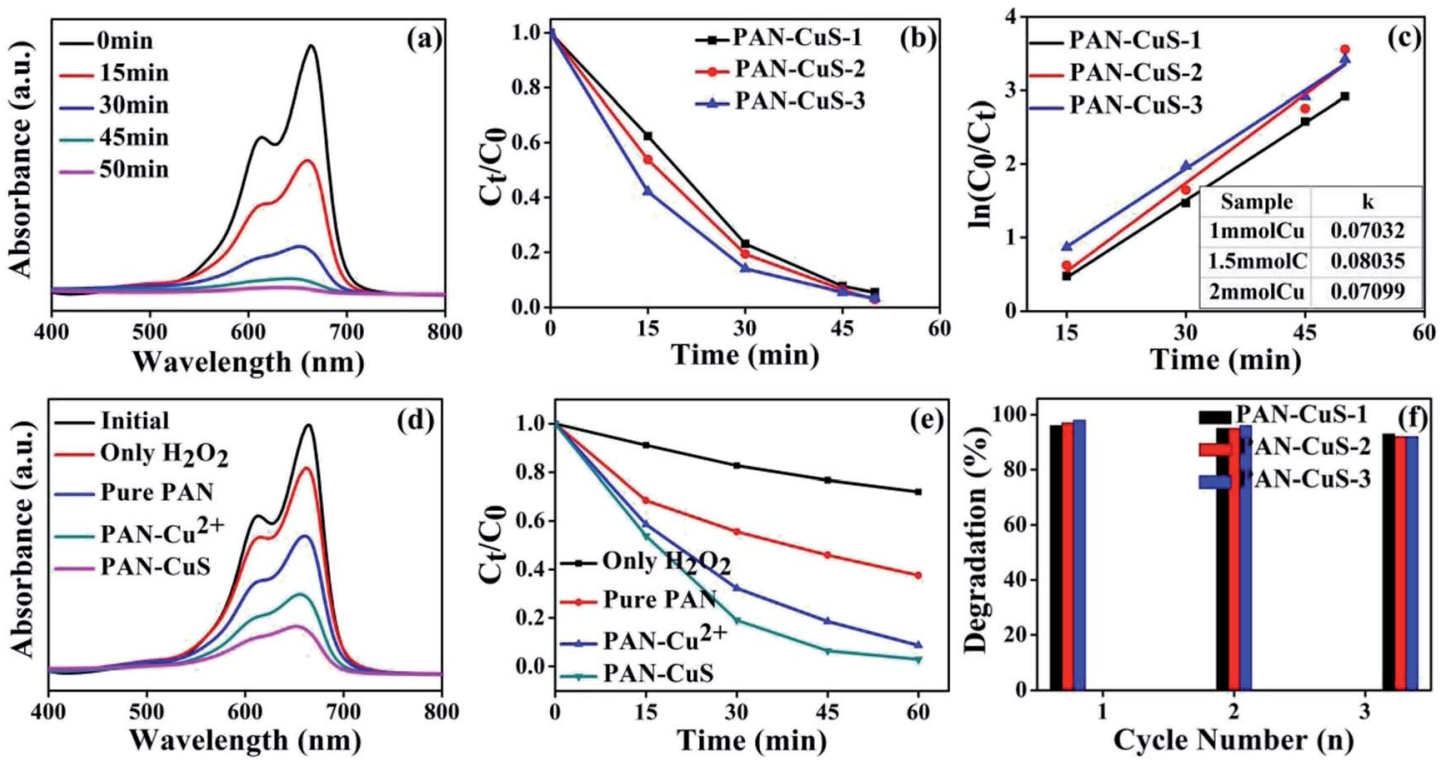

Fig. 3 (a) UV-Vis absorption spectra of MB solutions for different durations with PAN-CuS-2. (b) The MB degradation rates for different amounts of $\mathrm{Cu}^{2+}$. (c) Kinetic study for the degradation of $M B$; the inset shows the first order rate constant. (d) UV-Vis absorption spectra of MB degradation after 30 min for different samples: only $\mathrm{H}_{2} \mathrm{O}_{2}$, pure PAN, PAN-Cu${ }^{2+}, \mathrm{PAN}-\mathrm{CuS}$. (e) The $\mathrm{MB}$ degradation rates of different samples: only $\mathrm{H}_{2} \mathrm{O}_{2}$, pure PAN, PAN-Cu ${ }^{2+}$, PAN-CuS. (f) The \% degradation vs. cycle number suggesting the stability of PAN-CuS hierarchical structures toward MB degradation. All the measurements were carried out under UV.
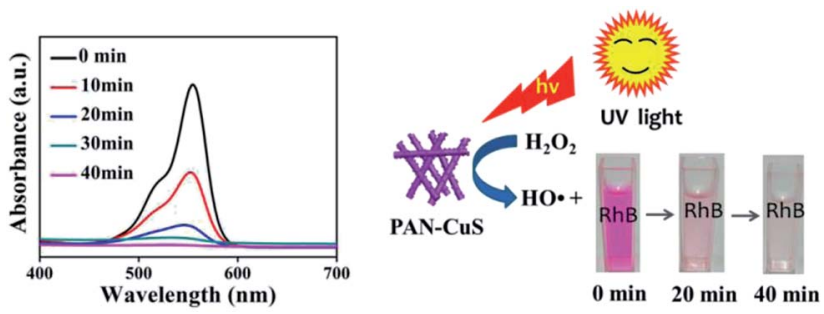

Fig. 4 (Left) UV-Vis absorption spectra of RhB solutions at different durations with PAN-CuS-2, (right) schematic of RhB degradation as a function of time after adding $1 \mathrm{~mL}$ of $\mathrm{H}_{2} \mathrm{O}_{2}$ with PAN-CuS hierarchical heterostructures under UV.

successfully realized close contact between the CuS nanosheets and the PAN fibers in the heterostructures. Such close contact is more effective for suppression of electron-hole recombination. Third, this PAN-CuS film can be easily recycled.

Recycling as well as maintaining high photocatalytic activity is a critical issue for the long-term use of catalysts in practical applications. Consequently, two factors need to be considered. ${ }^{46}$ (1) The ability of the catalyst to maintain its high activity over time is critical. It is known that the photocorrosion or photodissolution of photocatalysts may occur on the surface during the photocatalytic reaction. To test the stability of MB photodegradation on PAN-CuS films, we reused the catalyst three times. As shown in Fig. 3f, each experiment was performed under identical conditions and after three cycles, the photocatalytic activity of the PAN-CuS hierarchical heterostructures remained $94 \%$. (2) The ease with which the catalyst can be separated from solution must also be considered. In this study, the samples are nanofibrous and the films can be directly removed from the solution. Fig. S6† shows the SEM images of the samples after three cycles.

For studying the universality of the as-prepared PAN-CuS photocatalytic material, PAN-CuS-2 was also used to degrade the Rhodamine $\mathrm{B}(\mathrm{RhB})$ dye. The degradation result is exhibited in Fig. 4 (left). The decoloring degree was 53.7\%, 86.1\%, 93.9\%, and $98.4 \%$ after $10,20,30$, and $40 \mathrm{~min}$, respectively, indicating excellent degradation activity. On the basis of the above results, the dye degradation mechanism ${ }^{48}$ can be ascribed to the formation of hydroxide radicals in the presence of $\mathrm{CuS}$ and $\mathrm{H}_{2} \mathrm{O}_{2}$. The hydroxyl radicals, in turn, react with and degrade organic substrates such as RhB. Fig. 4 (right) shows the schematic of $\mathrm{RhB}$ degradation in the presence of $\mathrm{H}_{2} \mathrm{O}_{2}$ under UV light.

$$
\mathrm{H}_{2} \mathrm{O}_{2} \stackrel{\mathrm{CuS}}{\longrightarrow} \mathrm{HO}^{\bullet}
$$

$\mathrm{RH}+\mathrm{HO}^{\bullet} \rightarrow \mathrm{R}^{\bullet}+\mathrm{H}_{2} \mathrm{O}(\mathrm{RH}$ organic substrates such as $\mathrm{RhB})$

\section{Conclusions}

In summary, stringed "flowering branch" PAN-CuS hierarchical heterostructures were successfully fabricated through in situ sulfidation on electrospun fibers. This unique structure can provide abundant active sites, controllable morphology, and excellent charge separation, thus exhibiting enhanced photocatalytic activity in the decomposition of $\mathrm{MB}$ and $\mathrm{RhB}$ under UV light irradiation. Furthermore, these PAN-CuS hierarchical heterostructures with good flexibility can be easily recycled 
without a decrease in the photocatalytic activity. The results show that PAN-CuS-2 film has the strongest degradation effect, highlighting the role of CuS nanoflower assembly of nanosheets. It is expected that the development of such nanofibers by electrospinning with hierarchical heterostructures represents a very simple and cost-effective approach for the degradation of dye and will greatly promote their practical application to eliminate organic pollutants from wastewater.

\section{Conflicts of interest}

There are no conflicts to declare.

\section{Acknowledgements}

Thanks a lot for the support from the Natural Science Foundation of China (No. 21671205 \& 21701202), Henan Province (No. 15HASTIT003 \& 18A430034), Innovation Scientists and Technicians Troop Construction Projects of Henan Province (No. 164100510007 and CXTD2015018) and Zhengzhou University (No. 1421316035 and 2016xjxm258) and Program for Interdisciplinary Direction Team in Zhongyuan University of Technology, China.

\section{Notes and references}

1 A. Paracchino, V. Laporte, K. Sivula, M. Grätzel and E. Thimsen, Nat. Mater., 2011, 10, 456.

2 J. D. Xiao, J. Rabeah, J. Yang, Y. B. Xie, H. B. Cao and A. Brückner, ACS Catal., 2017, 7, 6198.

3 N. Shi, X. H. Li, T. X. Fan, H. Zhou, J. Ding, D. Zhang and H. X. Zhu, Energy Environ. Sci., 2011, 4, 172.

4 Y. J. Wang, D. G. Chen, Y. D. Wang, F. Huang, Q. C. Hu and Z. Lin, Nanoscale, 2012, 4, 3665.

5 D. Malwala and P. Gopinath, Environ. Sci.: Nano, 2015, 2, 78.

6 C. Fettkenhauer, X. C. Wang, K. Kailasam, M. Antonietti and D. Dontsova, J. Mater. Chem. A, 2015, 3, 21227.

7 Z. P. Wen, Y. L. Zhang, S. Guo and R. Chen, J. Colloid Interface Sci., 2017, 486, 211.

8 H. R Yang, H. X. Zhang, J. X. Peng, Y. Y. Zhang, G. Q. Du and Y. Fang, J. Colloid Interface Sci., 2017, 485, 213.

9 Q. Huang, S. B Deng, D. N. Shan, Y. J. Wang, B. Wang, J. Huang and G. Yu, J. Colloid Interface Sci., 2017, 488, 142.

10 Q. J. Xiang, J. G. Yu and M. Jaroniec, J. Am. Chem. Soc., 2012, 134, 6575.

11 R. Zhang, Q. Wang, J. Zhang, L. L. Yin, Y. Li, S. Yin and W. B. Cao, CrystEngComm, 2018, 20, 4651.

12 X. Chen, L. Liu, P. Y. Yu and S. S. Mao, Science, 2011, 331, 746.

13 T. W. Kim and K. S. Choi, Science, 2014, 343, 990.

14 J. Lee, S. Ham, D. Choi and D. J. Jang, Nanoscale, 2018, 10, 14254.

15 C. J Song, Y. Feng, W. D. Shi and C. B. Liu, CrystEngComm, 2016, 18, 7796.

16 K. Kamijyo, T. Takashima, M. Yoda, J. Osaki and H. Irie, Chem. Commun., 2018, 54, 7999.
17 X. Zong, G. P. Wu, H. J. Yan, G. J. Ma, J. Y. Shi, F. Y. Wen, L. Wang and C. Li, J. Phys. Chem. C, 2010, 114, 1963.

18 Z. J. Xu, T. Li, F. Y. Zhang, X. D. Hong, S. Y. Xie, M. D. Ye, W. X. Guo and X. y. Liu, Nanoscale, 2017, 9, 3826.

19 X. Y. Li, B. Wei, J. Wang, X. Li, H. J. Zhai and J. H. Yang, J. Alloys Compd., 2016, 689, 287.

20 A. A. Tahir, M. A. Ehsan, M. Mazhar, K. G. U. Wijayantha, M. Zeller and A. D. Hunter, Chem. Mater., 2010, 22, 5084.

21 P. Kar, S. Farsinezhad, X. J. Zhang and K. Shankar, Nanoscale, 2014, 6, 14305.

22 S. Adhikari, D. Sarkar and G. Madras, ACS Omega, 2017, 2, 4009.

23 J. Liu and D. Xue, J. Mater. Chem., 2011, 21, 223.

24 Y. Liu, M. X. Liu and M. T. Swihart, Chem. Mater., 2017, 29, 4783.

25 M. Saranya, R. Ramachandran, E. J. J. Samuel, S. K. Jeong and A. N. Grace, Powder Technol., 2015, 279, 209.

26 U. T. D. Thuy, N. Q. Liem, C. M. A. Parlett, G. M. Lalev and K. Wilson, Catal. Commun., 2014, 44, 62-67.

27 C. H. An, S. T. Wang, J. He and Z. X. Wang, J. Cryst. Growth, 2008, 310, 266.

28 C. Y. Wu, S. H. Yu, S. F. Chen, G. N. Liu and B. H. Liu, J. Mater. Chem., 2006, 16, 3326.

29 H. T. Zhu, J. X. Wang and D. X. Wu, Inorg. Chem., 2009, 48, 7099.

30 P. Roy, K. Mondal and S. K. Srivastava, Cryst. Growth Des., 2008, 8, 1530.

31 L. H. Wang, H. Chen, L. Xiao and J. H. Huang, Powder Technol., 2016, 288, 103.

32 L. W. Mi, W. T. Wei, Z. Zheng, Y. Gao, Y. Liu, W. H. Chen and X. X. Guan, Nanoscale, 2013, 5, 6589.

33 S. D. Sun, X. P. Song, C. C. Kong, S. H. Liang, B. J. Ding and Z. M. Yang, CrystEngComm, 2011, 13, 6200.

34 J. Xu, C. S. Lee, Y. B. Tang, X. Chen, Z. H. Chen, W. J. Zhang, S. T. Lee, W. X. Zhang and Z. H. Yang, ACS Nano, 2010, 4, 1845.

35 P. B. Liu, Y. Huang, J. Yan, Y. W. Yang and Y. Zhao, ACS Appl. Mater. Interfaces, 2016, 8, 5536.

36 Z. Wen and L. A. Wang, Mater. Chem. Phys., 2008, 109, 77.

37 H. Wang, J. R. Zhang, X. N. Zhao, S. Xu and J. J. Zhu, Mater. Lett., 2002, 55, 253.

38 W. W. He, H. M. Jia, X. X. Li, Y. Lei, J. Li, H. X. Zhao, L. W. Mi, L. Z. Zhang and Z. Zheng, Nanoscale, 2012, 4, 3501.

39 M. Nagarathinam, J. L. Chen and J. V. Vittal, Cryst. Growth Des., 2009, 9, 2457.

40 M. Xu, H. Y. Wu, P. M. Da, D. Y. Zhao and G. F. Zheng, Nanoscale, 2012, 4, 1794.

41 C. J. Niu, J. S. Meng, X. P. Wang, C. H. Han, M. Y. Yan, K. N. Zhao, X. M. Xu, W. H. Ren, Y. L. Zhao, L. Xu, Q. J. Zhang, D. Y. Zhao and L. Q. Mai, Nat. Commun., 2015, 6, 7402.

42 Q. Bai, F. C. Shen, S. L. Li, J. Liu, L.-Z. Dong, Z.-M. Wang and Y. Q. Lan, Small Methods, 2018, 1800049, DOI: 10.1002/ smtd.201800049.

43 H. Wang, L. F. Liang, X. J. Cheng, Y. M. Luo and S. Sun, Photochem. Photobiol., 2017, 94, 17. 
44 C. Y. Wu, S. H. Yu and M. Antonietti, Chem. Mater., 2006, 18, 3599.

45 X. P. Li, Y. N. Gao, L. Yu and L. Q. Zheng, J. Solid State Chem., 2010, 183, 1423.

46 M. Y. Zhang, C. L. Shao, J. B. Mu, X. M. Huang, Z. Y. Zhang, Z. C. Guo, P. Zhang and Y. C. Liu, J. Mater. Chem., 2012, 22, 577.
47 L. L. Li, P. N. Zhu, S. J. Peng, M. Srinivasan, Q. Y. Yan, A. S. Nair, B. Liu and S. Samakrishna, J. Phys. Chem. C, 2014, 118, 16526.

48 J. Kundu and D. Pradhan, ACS Appl. Mater. Interfaces, 2014, 6, 1823. 